\title{
Violencia familiar: un problema de salud pública en tiempos de pandemia
}

\author{
Jesús Alfredo Morales Carrero (iD) https://orcid.org/0000-0001-8533-3442
}

La convivencia familiar durante los últimos meses ha sufrido un deterioro significativo. Las relaciones entre padres e hijos, así como entre los demás miembros de este factor de socialización han estado mediadas en muchos de los casos por el maltrato físico y psicológico, motivado entre otras razones, por la permanencia prolongada, la escasa capacidad para fortalecer las relaciones interpersonales, la deficitaria inteligencia emocional y la dificultad para sobrellevar la tensión [1]. Como parte de los factores impulsores de esta ruptura del equilibrio familiar, los reportes indican que: la adopción de múltiples roles, el exceso de responsabilidades en unos miembros familiares más que en otros y la sobrecarga de la educación formal en los padres, ha obligado a destinar parte de su tiempo en la consolidación de las asignaciones de sus hijos, impulsando la violencia en sus diversas manifestaciones [2].

Según expone ONU-Mujeres [3] la pandemia ocasionada por el Covid-19 alteró el funcionamiento familiar y las relaciones humanas en general, entre otras razones porque el confinamiento "avivó la tensión y el estrés generados por preocupaciones relacionadas con la seguridad, la salud y el dinero; el aislamiento de las mujeres con compañeros violentos, creó la situación perfecta para ejercer un comportamiento controlador y violento en el hogar" (p.2). Esta posición es igualmente compartida por el Observatorio Nacional del Ciudadano (2020) [4], deja ver que la violencia familiar puede entenderse como "un incidente o patrón de incidentes de comportamiento de control, coercitivo, amenazante o degradante, en la mayoría de los casos por parte de una pareja o ex pareja, pero también de un familiar o cuidador" (p.4).
La Oficina de las Naciones Unidas contra la Droga y el Delito [5], informó que la violencia familiar durante el primer trimestre del año sufrió un incremento significativo, encabezando las estadísticas "el homicidio doloso de mujeres, el cual sufrió un alza de $5.1 \%$, siendo de un dato que refleja las implicaciones del confinamiento social; mujeres y niñas son las principales víctimas de la violencia homicida de pareja y de carácter familiar" (p.2).

Como resultado de este caos social, la emergencia de la violencia como fenómeno social multifactorial, se ha incrementado desde que se inició el confinamiento exigido como medida para evitar la propagación del Covid-19. Si realizamos una revisión minuciosa sobre los reportes que continuamente publican los diarios mundiales, nos encontramos que el confinamiento social se ha convertido en el catalizador de problemas al interior de la familia; parafraseando a Villate [2], los tiempos de crisis que afronta la humanidad en general deben entenderse como los más cruentos para la convivencia en sus diversas manifestaciones, pues la emergencia de estrés y tensión han provocado un aumento alarmante en los casos de maltratos contra la mujer. Según la autora las cifras de denuncias por violencia se muestran de la siguiente manera "Colombia 91\%, México 40\% y Perú registra 207 denuncias" (p.2). Los reportes indican que la estabilidad de la familia se encuentra vulnerada por muchas razones, entre las que se precisan: el temor de la mujer a denunciar, la desconfianza en los órganos de seguridad, la amenaza sistemática en las dimensiones física y psicológica, así como la dependencia socio-económica que impulsa a la víctima (mujer) a actuar de manera sumisa y pasiva.

\footnotetext{
${ }^{1}$ Docente del Departamento de Orientación y Psicología General de la Universidad de Los Andes, Venezuela. Investigador reconocido por el Programa de Estímulo a la Investigación (P.E.I) y por el Programa de Estímulo a la Docencia (P.E.D). Venezuela, Merida 5101. Correspondência para: JAM Carrero. E-mail: lectoescrituraula@gmail.com; jmoralescarrero@yahoo.com.
}

Como citar este artigo / How to cite this article

Carrero JAM. Violencia familiar. Un problema de salud pública en tiempos de pandemia. InterAmJ Med Health 2021;4:e202101017 
Según referencia el Informe de la Universidad de Anáhuac [6], la Organización Mundial de la Salud reporta que "cada año, aproximadamente 770 millones de mujeres son agredidas por su parejas y exparejas en el planeta" (p.5). Es fácil predecir que, por lo general, las víctimas de violencia de género además de sufrir daños físicos, psicológicos y morales, tienden por temor a ceder su voluntad y acceder a las pretensiones manipuladoras de su victimario, quien se vale del aislamiento sistemático, el desarrollo de un profundo sentimiento de culpabilidad que deriva en resignación, la adopción de una actitud dócil y la pérdida del sentido crítico, lo que genera "el miedo que lleva a la obediencia, a la sumisión, en la persona atacada" [7].

Estos factores de riesgo han tomado fuerza durante la pandemia debido a que las políticas de confinamiento, solo han estimado el recrudecimiento de las restricciones, lo que ha ocasionado "la limitación en los movimientos, la reducción de las interacciones entre la comunidad, el cierre de servicios y el empeoramiento de las condiciones socioeconómicas, factores que han ocasionado un incremento en las posibilidades de sufrir violencia doméstica [8]. Pont [9] afirma que "antes de comenzar la pandemia y la aplicación radical de las medidas de confinamiento social un $30 \%$ de las mujeres a nivel mundial, habían sufrido violencia tanto física como sexual" (p.6). Es preciso indicar que, esta cifra con seguridad se ha incrementado de tal manera que se estima que dos de cada tres mujeres son víctimas de sujetos controladores, que doblegan la personalidad y reducen la estima propia, como medios para ejercer dominio total de su voluntad.

Esta subordinación lleva a la mujer a asumir una actitud tolerante frente a los abusos que se le infligen, los cuales pueden adquirir diversos modos, entre los que se precisan "el daño o sufrimiento físico, sexual o psicológico, incluyendo amenazas, coerción, privación de libertad y el abuso en sus múltiples manifestaciones" [10]. Por lo general, la víctima frente a este círculo de la violencia, se ve neutralizada posibilitándole al hombre el poder para ejercer control y dominación total sobre su persona. Este proceder se debe entre otras razones, a la consolidación de un sistema que ha legitimado la violencia contra la mujer, a quien se le ha infravalorado su posición social, así como desvalorizado su rol protagónico en las actividades domésticas [11], condiciones socio-históricas que se le atribuyen significativamente a la justificación o normalización que esta le ha otorgado su trabajo en el hogar y a su función social en general.

González [12] refiriéndose al reporte de violencia que experimentan otros escenarios del mundo, reitera que la mujer constituye el blanco de los abusos en sus distintas manifestaciones; las cifras indican que "casi una cuarta parte de las estudiantes en EE.UU afirman haber sido víctimas de agresión sexual y de comportamientos impropios, mientras que en el África la violencia entre parejas suponía una realidad para el $65 \%$ de las mujeres" (p.8). Según expone García [13], la violencia en países como España ha estado asociada con el machismo que reforzado por el confinamiento, se ha consolidado como un binomio peligroso para la integridad de la mujer. Según la autora el año en curso refleja el asesinato de "dos mujeres por sus parejas, tres feminicidios familiares de hijos que asesinaron a sus madres durante el confinamiento, además de otros dos casos aún por resolver y un feminicidio no íntimo. Ocho mujeres en total fueron asesinadas en España". Este panorama se asemeja a la realidad de Bolivia, en la que según Sosa [14] se han dado "cuatro femicidios, 1200 casos de violencia y 33 casos de violaciones a menores de edad" (p.2).

Lo anterior puede interpretarse como el resultado de una sociedad machista, que asume la superioridad del hombre sobre la mujer, adjudicándose su propiedad y el uso legítimo de la fuerza, las humillaciones sistemáticas, el sometimiento y las agresiones permanentes, como conductas en las que subyace la degradación de la autoestima, dejándolas en un estado de vulnerabilidad que les imposibilita reaccionar a tiempo para salvar sus vidas. Para Hirigoyen [7], el hombre violento se vale de "comportamientos seductores, la imposición y la amenaza, hasta lograr aplastar a su víctima. Esto usualmente crea las condiciones para que se perpetre el asesinato de la víctima" (p.56). Uno de los casos emblemáticos en el que el machismo tiene mayor presencia, refiere a la sociedad mexicana, en la que su modus operandi viene dado por "diversas creencias, culturas o situaciones que llevan a cierta parte de la población a discriminar a la mujer, no considerar su opinión y en muchas ocasiones violar sus derechos e integridad" [6].

Este proceso sistemático de violentar la integridad de la mujer, cuenta con particularidades arraigadas en la desigualdad social. De allí que sea necesario precisar que sus resultados se manifiestan a nivel familiar mediante los siguientes rasgos "abuso psicolóngico y/o emocional, abuso físico o sexual, abuso financiero o económico, acoso y acecho, abuso en línea o digital"[15]. Por lo general, estos factores de riesgo que experimenta la familia a nivel mundial, son fomentados por otros problemas sociales vinculados con la profundización de la disfuncionalidad 
familiar, la legitimación de la superioridad del hombre sobre la mujer y el maltrato sistemático que da lugar "al comportamiento sumiso y dócil en exceso, ocasionando que los procesos de intervención sean más complejos de aplicar" [16].

Según el reporte de la Universidad de Anáhuac [6], la pandemia generada por el Covid-19 ha ocasionado daños físicos y psicológicos en la familia, pero con mayores repercusiones en la mujer, pues la prolongada convivencia ha posibilitado las condiciones para que se perpetren abusos de poder, ocasionando el ejercicio del "dominio, el sometimiento, el control y la agresión de manera física, verbal, psicológica, patrimonial, económica y sexual dentro o fuera del domicilio familiar" (p.4). Como una manera de tipificar la violencia de género, los organismos mexicanos de atención a la víctima tipificaron los principales rasgos de la violencia tanto física como psicológica. La primera estima dentro de su caracterización "los moretones, raspaduras, cortaduras, fracturas, quemaduras, luxaciones, lesiones musculares, golpes en la región de la cabeza, en la cara; mientras que la segunda "autoestima baja, sentimientos de miedo, ira, vulnerabilidad, tristeza, humillación, desesperación, entre otros" (p.10).

Los datos aportados por Pont [9] indican que la violencia de género experimentó una alarmante escalada a partir del mes de marzo; por ejemplo, países como Francia sufrieron un incremento de $30 \%$ en abusos sexuales y maltratos a mujeres; por su parte en Argentina la data es de un poco más de 39\%, mientras que países como Singapur las asociaciones que manejan los registros de violencia indicaron que solo en un mes se recibieron 612 denuncias de víctimas solicitando ayuda. Por su parte Reino Unido pese a la existencia de programas de atención a víctimas de agresión doméstica, las cifras aumentaron un $25 \%$. A pesar de las alarmantes cifras que confirman los efectos del confinamiento prolongado, el caso de Somalia constituye uno los más representativos, pues se estima que durante los últimos meses las mujeres entre 15 y los 49 años de edad, han sufrido mutilaciones, maltratos físicos y psicológicos, abusos sexuales, entre otros.

Al respecto Romero [17] indica que las medidas sugeridas por la Organización de Naciones Unidas con respecto al Covid-19 incluyen prioritariamente el diseño particularizado de medidas de protección a las mujeres, dada la elevada cantidad de denuncias que en cada país de América Latina se dan a diario. Según la autora, la violencia se ha convertido en un problema de salud pública que afecta no solo a la mujer sino a los demás miembros de la familia, en quienes se da el incremento de los niveles de estrés y la tensión en las relaciones interpersonales. Como una medida paliativa generalizada, los gobiernos de Argentina, Colombia y Chile han asumido la necesidad de proponer políticas públicas que eduquen a la familia, fortalezcan los vínculos que determinan la convivencia y dimensionen los lazos comunicativos como requerimientos para minimizar el círculo de acción del agresor.

En opinión de Fuentes [18] el sustento de la violencia de género se debe, entre otras razones, a la presencia de "mucha tensión, incertidumbre, dificultades financieras y problemas psicológicos, motivos que hacen pensar que en familias donde ya existía violencia intrafamiliar esta se acentuó" (p.4). A esto se agrega las implicaciones de las masculinidades construidas a lo largo de la historia, en las que el rol de cuidar, cooperar con las labores domésticas y salvaguardar la integridad de la familia no representa una prioridad para el hombre, lo que ha traído como consecuencia la agudización de la desigualdad de género y la asimetría de poder en favor de la figura masculina.

Para el Banco Interamericano de Desarrollo [19], la violencia doméstica requiere especial atención por parte de las entidades gubernamentales encargadas de velar por el bienestar de la familia. Esto sugiere, ofrecer programas de ayuda, orientación y asesoramiento que bloqueen la expansión de comportamientos machistas; a esto se agrega, la necesidad de diseñar dispositivos jurídicos que regulen al menos temporalmente el acceso al consumo de alcohol. La institución asegura que "las pruebas internacionales demuestran que el abuso de alcohol está presente en al menos un tercio de los casos de abuso sexual" (p.2).

En países como Argentina, el confinamiento prolongado constituyó junto al consumo de sustancias psicoactivas, detonantes de los casos de violencia; para el mes de marzo el porcentaje era de $36 \%$, mientras que en mayo la cifra superó los $47,7 \%$, ocasionando en las víctimas un estado de indefensión, responsable de estados profundos de depresión, desesperación y propensión a desarrollar ideas e intentos suicidas [14]. Parafraseando a Expósito [16], las víctimas de violencia familiar tienden a desarrollar el síndrome de estrés postraumático, ocasionando la emergencia de profundas sensaciones de ansiedad, depresión, estimada baja y dificultades para integrarse socialmente.

Por su parte, Isaza [20] refiriéndose a los reportes del Observatorio Colombiano para las Mujeres, registró cerca de 1674 casos de violencia intrafamiliar, discriminados 
cualitativamente de la siguiente manera: parte de las víctimas contaban con antecedentes de violencia perpetrada por su pareja; el consumo de sustancias psicoactivas se reflejó como un factor común que exacerbó las conductas violentas contra los miembros de la familia, otros casos, en modo significativo manifestaron sentir la tensión económica como un impulsor de frustración, agresión e inestabilidad emocional. Como parte de las medidas ofrecidas, el Estado colombiano ha creado programas de atención a las víctimas, abordando de manera focalizada los problemas asociados con el manejo de la incertidumbre y el caos, las implicaciones de los cambios bruscos y la adaptación a la recurrente tensión social, como detonantes de la violencia: sexual, económica, psicológica, física y moral.

Desde la perspectiva de Llínás [21], los datos aportados por la Medicina Legal colombiana indican que la escalada de la violencia de género alude a manifestaciones estructurales e históricas, pues es en la familia donde se reproducen no solo maltratos físicos y psicológicos, sino expresiones de poder sustentadas en la falta de autonomía económica de la mujer para sufragar sus propias necesidades. Los reportes indican que la violencia contra la mujer en Colombia alcanzó "durante el primer trimestre del año 2020, los 11.000 casos; las denuncias por agresión superaron las 2.209 en comparación al año 2019, en el que el tope fue de 964" (p.2). Además, según cifras de la Fiscalía el feminicidio alcanzó los 19 casos, a lo que se agrega 3.069 denuncias asociadas con violencia intrafamiliar [21].

Esta situación no discrepa en países como Panamá, que junto a "Brasil, México, Argentina, Perú, El Salvador y Bolivia representando el $81 \%$ de los casos globales" [22]. En estos lugares la violencia involucra abusos sexuales a niñas y jóvenes, quienes dada la paralización de las actividades educativas, ocupacionales y económicas, se han visto obligadas a convivir con su agresor, incrementado las posibilidades de ser asesinadas o sometidas a daños irreversibles, incluyendo el embarazo en adolescentes como resultado de las recurrentes violaciones de las que son objeto. En tal sentido, la vida al interior de los hogares se ha convertido en un espacio de castigo y abuso. Los testimonios de niñas a lo largo de América Latina reiteran haber sido abusadas sexualmente por quienes hacen las veces de padre $u$ otras figuras masculinas con las que conviven a diario se convierten en un acto común. Estos abusos físicos y psicológicos se discriminan de la siguiente manera "de maltrato físico se mantiene estable respecto a 2018 (de un 14\% al 15,1\%); el psicológico ha escalado del $7,6 \%$ al $14,4 \%$. También repunta el abuso sexual (del $3,8 \%$ al $4,8 \%$ ), y el abandono (del 1,7\% al 3,6\%)" [14].

En el caso de Venezuela, el diario Efecto-Tocuyo indica que el Centro de Justicia y Paz (2020) registró cerca de 196 casos de violencia de género de los cuales 190 se dieron durante el periodo marzo-noviembre, tiempo en el que el gobierno venezolano radicalizó las medidas de confinamiento social. Para esta organización el asedio de las mujeres en el país indica que se está frente a una emergencia feminista, pues "se reportó que entre abril y junio se dieron más de 43 femicidios" (p.1). Al respecto la Agencia EFE Héctor Pereira en su informe (2020) [23] indican que este año las cifras han sido alarmantes, un poco más de "200 mujeres han sido asesinadas durante la pandemia en el país petrolero" (p.5). Por la diversidad en los modos como se manifiesta la violencia en el país, es estimada por los especialistas como un espacio en el que tiene presencia las mil formas de violencia de género o de la violencia machista. Según indica Proyecto Migración Venezuela, el país reportó "Más de 2.000 casos de violencia de género" (p.7). Para la organización la violencia contra la mujer venezolana en países vecinos como Colombia, subió a 239 casos, más alarmante aún si se discrimina los efectos de la violencia durante el último tercio del 2020, los cuales se discriminan de la siguiente manera: 1.257 fueron por violencia física; 719, por violencia sexual; 595, por negligencia o abandono; y 89, por violencia psicológica.

Es oportuno aclarar que, si bien es cierto la violencia familiar durante el confinamiento social se ha reportado con especial énfasis en la mujer, esta ha representado solo un segmento de este fenómeno social, pues según el Observatorio Nacional del Ciudadano [15], el maltrato se extiende a otros miembros de la familia, caso específico infantes "que suelen verse atrapados en relaciones de violencia y abuso; los niños son testigos de aproximadamente tres cuartas partes de esos incidentes abusivos. Aproximadamente la mitad de los niños y las niñas de esas familias han sido golpeados" (p.5).

Otros reportes estadísticos del año en curso precisan que la violencia familiar se convirtió en uno de los problemas de salud pública frente a los cuales el Estado, no ha logrado mayor intervención; al menos eso lo demuestran las siguientes cifras: 


\begin{tabular}{|c|c|c|}
\hline País & Año & Cifras \\
\hline México & 2020 & Aumentó 30\% violencia familiar \\
\hline Chile & 2020 & Aumentó 70\% violencia intrafamiliar \\
\hline \multicolumn{3}{|c|}{ Fuente: ONU-Familia, 2020 } \\
\hline Singapur/Chipre & 2020 & Aumentó del 30\% violencia familiar \\
\hline Australia & 2020 & Aumentó 40\% violencia familiar \\
\hline \multicolumn{2}{|c|}{ Fuente: ONU-Mujeres, 2020 } \\
\hline EE.UU & 2021 & Aumentó del 25\% \\
\hline Sudáfrica & 2021 & violencia sexual más la \\
\hline Fuente: Corthier, 2021 &
\end{tabular}

Elaboración: Morales, 2021

\section{Discusión en torno a la violencia familiar}

El cumplimiento de la normas de bioseguridad a nivel mundial ha dejado a un lado el tratamiento de problemas igual o más graves asociados con la violencia de género. Las cifras indican que al menos dos de tres mujeres en el mundo sufren de maltratos físicos y psicológicos, que se manifiestan de modo diverso. Para algunos, es el resultado de patrones aprendidos y legitimados por la cultura, y muchas veces normalizado por la mujer, en quien el victimario desarrolla un estado de indefensión y desesperanza que da lugar a la perpetración de los más aberrantes abusos [7]. Según indica el Observatorio Nacional del Ciudadano [4], el confinamiento se ha convertido en un agravante de la violencia de género, dado que ha exacerbado "comportamientos de control, el uso de la fuerza, la coerción, conductas amenazantes y degradantes, el abuso psicológico y/o emocional, abuso físico o sexual, abuso financiero, acoso y acecho, abuso en línea o digital" (p.4).

Por lo general, la violencia familiar se encuentra motivada no solo por el maltrato físico y psicológico, sino por "la imposición del poder en de quien la ejerce para regular la conducta de quien es objeto de dicha acción y omisión" [10]. Este proceder propio del victimario procura, entre otras cosas, la incapacidad de la mujer para tomar decisiones, la anulación de su voluntad y la degradación de la autoestima, en un intento sistemático por debilitar cualquier pretensión de romper con el círculo de violencia en el que se encuentra inmersa. Como parte de las implicaciones de estas relaciones nocivas, se han incrementado los reportes de femicidio a nivel mundial, pues la pandemia generada por el Covid-19 ha obligado a las víctimas a convivir con sus victimarios durante tiempos prolongados que dan lugar sentimientos de frustración, estados de tensión y estrés que asumidos como factores 
de riesgo, exacerban las condiciones para la violencia contra la mujer, quien en su intento por proteger a sus hijos se convierte en receptora de los más crueles actos de abuso y maltrato.

Este proceder del maltrato no discrimina entre el abuso hacia su pareja y quienes integran su núcleo familiar (hijos), a quienes directa e indirectamente los involucra en el acto violento y sus implicaciones, ocasionando daños psicológicos y emocionales que, en la mayoría de las veces de convierten en factores de riesgo que derivan en la reproducción futura de comportamientos violentos sobre terceros. Por lo general, individuos provenientes de hogares violentos tienden a desarrollar según expone Expósito [16] una serie de psicopatologías, entre las que se precisan "alteraciones de la personalidad, la adopción de patrones desadaptativos de resolución de problemas familiares, carácter agresivo, falta de control de la ira por experiencias de malos tratos" (p.20).

Por otra parte, es preciso indicar que la violencia familiar como fenómeno social multifactorial o multicausal, ha tomado especial auge durante lo que va del año 2021, entre otras razones por "menos del 40 por ciento de las mujeres que sufren violencia buscan ayuda de algún tipo o denuncian el delito. Menos del 10 por ciento de estas mujeres que buscan ayuda recurren a la policía" [3]. Según reporta este organismo, las víctimas que no logran activar mecanismos jurídicos que garanticen su protección, lo hacen por temor a sus victimarios, a lo que se une la deficitaria actuación judicial en gran parte de los países del mundo, razones por las cuales se elevan los índices de impunidad y, por lo tanto, la vulneración de la integridad de la mujer.

En consecuencia, minimizar la violencia de género requiere de un esfuerzo institucional, en el que se estime el desarrollo de competencias emocionales y habilidades sociales que permitan la convivencia sana y pacífica. Además, se hace perentorio la formulación de políticas públicas de atención integral a la familia, a través de las cuales educar para la vida, para el fortalecimiento de las relaciones interpersonales y el reconocimiento del otro en condiciones de igualdad y respeto. Esto sugiere, la aplicabilidad de mecanismos jurídicos efectivos que recuperen la confianza de la mujer en la activación de medidas de protección a su integridad física y psicológica.

En atención a este problema de salud pública, organismos internacionales como el Centro Global para el desarrollo ha propuesto una serie de medidas o sugerencias para atender a las víctimas de violencia familiar, entre las que se precisan "programas de ayuda alimentaria, monetaria, de formación y acceso a la tecnología deben priorizar a las mujeres, así como servicios de protección social y salud, incremento del poder económico y adquisitivo" [1]. Esto con el propósito de romper con los mecanismos de dependencia que hacen más vulnerables a la mujer frente a sus victimarios; de allí la iniciativa de la Organización de Naciones Unidas-Mujeres, en acercar durante el confinamiento social, una serie de acciones de intervención estratégica en áreas específicas como "atención y apoyo a las sobrevivientes, la gestión clínica de las violaciones, y el apoyo psicosocial y para la salud mental, que ayude a la víctima a superar los traumas y secuelas ocasionadas por la violencia" (p.3).

En función de lo anterior, se precisan algunas medidas asumidas por los entes gubernamentales y los colegios de profesionales en materia de atención a una vida libre de violencia, entre las que se mencionan:

1- Creación de canales de comunicación que faciliten la denuncia de casos de violencia, así como el seguimiento a las víctimas. Esto con el propósito de desarticular el efecto sistemático y nocivo que ejerce la violencia sobre la integridad de la mujer.

2- Consolidar cuerpos interdisciplinarios que aborden las consecuencias del maltrato psicológico y físico, así como brigadas de protección familiar que ofrezcan asesoramiento integral.

3- Fortalecimiento de las políticas públicas con enfoque a la atención a las diversas formas de discriminación. Esto incluye, entre otras cosas, programas asociados con "el apoyo económico y paquetes de estímulo acordes con la gravedad y la magnitud del reto que reflejen las necesidades de las mujeres que se enfrentan a diversas formas de violencia" [3].

4- Uso de las redes tecnológicas para potenciar los canales de atención, apoyo psicosocial y asesoramiento en línea. Ello permitirá la inclusión a más víctimas, así como desplegar acciones de intervención preventiva en espacios vulnerables.

5- Diseño de políticas públicas con enfoque de género y ejecución local, con el propósito de promover la inclusión y el trato igualitario como condiciones que propicie mayores niveles de independencia y autonomía en la mujer.

6- Monitoreo sistemático, constante y metódico de feminicidios, además de reportes periódicos de víctimas y victimarios, con el objetivo de ofrecer asesoramiento integral e intervención-preventiva [5]. 
7- Creación de redes de protección al grupo familiar. Estas ofrecen acompañamiento a las víctimas desde la dimensión jurídica hasta tratamiento en materia de salud integral, que involucra proveer las estrategias socioafectivas y las competencias emocionales necesarias para afrontar las consecuencias del maltrato al que fueron sometidas [15].

En síntesis, se puede afirmar que los reportes sobre violencia familiar sufrieron un incremento acelerado a partir de la aplicación radical de las medidas de confinamiento social. Frente a este desafío, gran parte de los gobiernos mundial han debido reformular, reconducir y estimar las políticas públicas en materia de atención integral a la familia, ofreciendo asesoramiento psicosocial como medida paliativa de cara a una pandemia que atenta contra el bienestar y la calidad de vida de la ciudadanía en todas sus dimensiones. De allí, la tarea del Estado en construir espacios y programas que maximicen la inclusión y enfrente "la macha negra de esta pandemia, reflejo de nuestros valores, nuestra resiliencia y nuestra humanidad compartida, que se ven ahora amenazados" [3].

\section{REFERENCIAS}

1. Avendaño S. Cepaz ha atendido 196 casos de violencia de género en Venezuela este 2020 [cited 2020 Nov 11]. Availabe from: https://efectococuyo.com/la-humanidad/ cepaz-ha-atendido-196-casos-de-violencia-de-genero-envenezuela-este-2020/.

2. Banco Interamericano de Desarrollo. Confinamiento y violencia dentro del hogar: Cuando el peligro está en casa [cited 2020 Mai 05). Availabe from: <https://www. diarioconstitucional.cl/2020/05/20/confinamiento-yviolencia-dentro-del-hogar-cuando-el-peligro-esta-encasal>.

3. Bourdieu P. La dominación masculina. Barcelona: Editorial Anagrama; 2020.

4. Corthier I. Violencia de género [cited 2021 ab 03]. Availabe from: <https://elpais.com/planeta-futuro/2021-01-25/ nace-una-iniciativa-para-cuantificar-el-enorme-dano-dela-covid-19-a-las-mujeres.html>.

5. Expósito F. Violencia de género. Revista Mente y Cerebro ( não encontrei a abreviação), 2011;48: 20-25.
6. Fuentes $A$. Violencia de género: la pandemia que viven las mujeres en cuarentena [cited 2020 Junho 06] Availabe from: <https://www.uc.cl/noticias/violencia-de-genero-lapandemia-que-viven-las-mujeres-en-cuarentena/>.

7. García N. Coronavirus y violencia de género: un binomio peligroso. Ayuda en Acción [cited 2020 abril 04]. Availabe from: <https://ayudaenaccion.org/ong/blog/mujer/ coronavirus-violencia-genero/>.

8. González A. Ante el aumento de la violencia doméstica por el coronavirus, Guterres llama a la paz en los hogares. Noticias Organización de Naciones Unidas [ cited 2020 abril 04]. Availabe from: <https://news.un.org/es/ story/2020/04/1472392>.

9. Hernández I. Violencia de género. Una mirada desde la sociología. La Habana: Editorial Científico-Técnica; 2014.

10. Hirigoyen M. El acoso moral. El maltrato psicológico en la vida cotidiana. Barcelona: Editorial Paidós; 1999.

11. Informe de la Universidad de Anáhuac. Violencia doméstica y de género en tiempos de COVID-19 [cited 2020]. Availabe from: <https://www.anahuac.mx/mexico/ noticias/Violencia-domestica-y-de-genero-en-tiempos-deCOVID-19>.

12. Isaza P. Violencia intrafamiliar en aumento por la pandemia. Agencia de noticias UPB - Medellín [cited 2020 maio 05]. Availabe from: <https://www.upb.edu.co/es/ noticias/violencia-intrafamiliar-aumenta-por-aislamiento>.

13. Llinás P. La premisa "quédate en casa" le cuesta la vida a mujeres de Colombia [cited 2020 abril 04]. Availabe from: $<$ https://www.cinep.org.co/Home2/component/k2/796-lapremisa-quedate-en-casa-le-cuesta-la-vida-a-mujeres-decolombia.html>.

14. Observatorio Nacional del Ciudadano.El confinamiento como agravante de la violencia familiar [cited 2020]. Availabe from: <https://onc.org.mx/uploads/ViolenciaFamiliar.pdf>. 15. Observatorio de Seguridad Secretaría de Seguridad y Justicia-Colombia [cited 2020]. Availabe from: <https:// www.cali.gov.co/observatorios/publicaciones/156979/ informes-especiales/>.

16. Oficina de las Naciones Unidas Contra la Droga y 
el Delito. Monitoreando la violencia contra las mujeres durante el confinamiento por la pandemia del COVID-19 [cited 2020]. Availabe from: <https://www.unodc. org/documents/mexicoandcentralamerica/2020/CdE/ Monitoreando la violencia contra las mujeres durante el confinamiento por la pandemia del COVID-19.pdf > .

17. Organización de Naciones Unidas-Mujeres. Violencia contra las mujeres: la pandemia en la sombra [cited 2020]. Availabe from: <https://www.unwomen.org/es/news/ stories/2020/4/statement-ed-phumzile-violence-againstwomen-during-pandemic>.

18. Pereira $\mathrm{H}$. Venezuela y sus mil formas de violencia de género [cited 2020 Nov 11]. Availabe from: <https://www. efe.com/efe/america/sociedad/venezuela-y-sus-mil-formasde-violencia-genero/20000013-4403658>.

19. Plan Internacional. Covid-19: alarmante aumento de la violencia contra las niñas y las mujeres en América Latina y El Caribe [cited 2020 maio 05]. Availabe from: <https:// plan-international.org/es/press-release/2020-05-19-covid19-alarmante-aumento-de-la-violencia-contra-las-ninas-ylas-mujeres>.

20. Pont E. La violencia contra las mujeres: la consecuencia invisible de la pandemia. La Vanguardia [cited 2020 junho 06]. Availabe from: <https://www.lavanguardia.com/vida/ junior-report/20200602/481576160749/violencia-generoaumenta-coronavirus-pandemia.html>.

21. Proyecto Migración Venezuela. Se dispara la violencia de género contra los migrantes [cited 2020]. Availabe from: $<$ https://migravenezuela.com/web/articulo/se-dispara-laviolencia-de-genero-contra-los-migrantes/2112>.

22. Romero M. ONU: violencia machista durante el confinamiento ha tenido un repunte "horroroso" [cited 2020 abril 04]. Availabe from: <https://www.france24. com/es/20200406-repunte-violencia-machista-cuarentenacoronavirus-mujeres-victimas >.

23. Salgado R. La pandemia del coronavirus aumenta el riesgo de violencia de género hacia mujeres y niñas desplazadas y apátridas [cited 2020 abril 04]. Availabe from: $<$ https://www.acnur.org/noticias/press/2020/4/5e9d5f5d4/ la-pandemia-del-coronavirus-aumenta-el-riesgo-deviolencia-de-genero-hacia.html>.
24. Sosa M. La crisis del coronavirus [cited 2020 maio 05]. Availabe from: <https://elpais.com/sociedad/2020-05-05/ violencia-durante-el-confinamiento-mi-padre-me-pega-yme-toca-me-siento-atrapada.html>.

25. Villate L. (16-04-20). El confinamiento incrementó la violencia intrafamiliar, ¿por qué? [cited 2020 abril 04]. Availabe from: <https://www.uoc.edu/portal/es/ news/colombia/noticias/199-confinamiento-violenciaintrafamiliar.html>. 